\title{
Importancia de las imágenes espaciales en el rendimiento académico en
}

\section{Economía}

\section{The importance of spatial imagery in the academic performance in Economics}

\author{
Jesús A. Dopico, María Ángeles González, Diego Campos-Juanatey \\ Universidade da Coruña
}

\begin{abstract}
Resumen
Con el objetivo de analizar la influencia de la habilidad espacial, la habilidad de rotar imágenes y el género, en el rendimiento en Economía, a un grupo de 119 estudiantes de Administración y Dirección de Empresas, se le aplicó la Measure of the Abililty to Form Spatial Mental Imagery, y el test Mental Rotation Test. Se realizó un examen sobre un modelo gráfico ampliamente utilizado en la enseñanza de la macroeconomía, y se obtuvo la calificación en la materia de Política Económica. Los participantes con alta habilidad para rotar imágenes obtuvieron puntuaciones significativamente superiores, tanto en la prueba de gráficos como en la materia evaluada, a los participantes con baja habilidad de rotación de imagen. Ambas puntuaciones de rendimiento fueron significativamente más altas en las mujeres que en los hombres. Por último, la puntuación obtenida en la prueba de gráficos por los individuos con alta habilidad de imagen espacial fue significativamente superior a la obtenida por los estudiantes con baja habilidad espacial. Palabras clave: imagen espacial, rotación mental, género, rendimiento académico, economía.
\end{abstract}

\begin{abstract}
In order to analyze the influence of spatial ability, mental rotation ability and gender, in the performance in Economics, the Measure of the Abililty to Form Spatial Mental Imagery, and the Mental Rotation Test, were applied to a group of 119 students of Business Administration. Their performance on a test about a graphic model widely used in the teaching of macroeconomics, and their examination results in Economic Policy, were obtained. Participants with high mental rotation ability scored significantly higher than those with low mental rotation ability, both in the graph test as in the assessed subject. Both performance scores were significantly higher in women than in men. Finally, the score obtained in the graph test by individuals with high spatial image ability was significantly higher than the score obtained by students with low spatial ability.

Keywords: spatial image, mental rotation, gender, academic achievement, economics.
\end{abstract}

\section{Introducción}

La relación entre las habilidades de imagen de los estudiantes y su rendimiento académico ha sido estudiada en diferentes ámbitos artísticos y científicos. Especialmente, se ha hecho hincapié en la existencia de una relación entre la rotación mental y la habilidad espacial y los resultados en ciencia, ingeniería, tecnología y matemáticas (campos identificados con el acrónimo anglosajón STEM; ver Khine, 2017, para una revisión reciente en este ámbito).

Entre otros ejemplos, Guillot, Champely, Batier, Thiriet y Collet (2007) señalan que el hecho de poseer una alta habilidad espacial, medida por estos autores a través del test MRT (Mental Rotation Test; Vandenberg y Kuse, 1978), puede considerarse un predictor del éxito en el aprendizaje de anatomía. Yoon y Min (2016) encontraron correlaciones significativas entre la habilidad espacial de los estudiantes de cursos de ciencia atmosférica (medida a través del Revised Purdue Spatial Visualization Tests: Visualization of Rotations; Revised PSVT: R; Yoon, 2011) y todas las medidas del rendimiento del curso. Cui, Wilson, Rockhold, Lehman y Lynch (2017) observaron que las puntuaciones de los estudiantes con alta habilidad de rotación mental (medida a través del MRT) en un test de conocimientos de anatomía, eran significativamente superiores a las de los estudiantes con baja habilidad de rotación, cuando en las clases se usaban imágenes en dos dimensiones.

En Economía, a pesar de que, como en otras titulaciones de ciencia, muchos de los conceptos son presentados con la ayuda de medios visualizados espacialmente (como diagramas, gráficos y mapas), apenas existen estudios específicos sobre la relación entre las habilidades de imagen y el rendimiento académico. Indirectamente, algunos autores han analizado la eficacia o idoneidad de diferentes métodos que en mayor o menor medida están vinculados con las imágenes (material espacial como los gráficos), a la hora de explicar principios económicos (Cohn, Cohn, Bach y Bradley, 2001; Reingewertz, 2013).

Cohn y cols. (2001) llevaron a cabo dos experimentos para comprobar si los estudiantes de Principios de Economía se beneficiaban de la utilización de gráficos en la clase. En uno de los experimentos, el uso de gráficos como complemento del material verbal pareció reducir el aprendizaje, mientras que en el segundo experimento, no se encontraron diferencias significativas entre el aprendizaje de los estudiantes a los que presentaron gráficos en las clases y aquellos a los que no. Al margen de que cierta ausencia de control experimental sobre la comprensión de los gráficos pudo 
haber influido en los resultados, los autores señalan que puede haber circunstancias y características de los estudiantes que limiten el beneficio que se obtenga con dicho método.

Reingewertz (2013) afirma también que incluso los modelos macroeconómicos más simples, como el modelo IS-LM podrían resultar demasiado complejos para algunos estudiantes.

Dopico, González y Campos-Juanatey (en prensa) estudiaron si la preferencia y dificultad asociada a diferentes métodos explicativos en Economía, estaba relacionada con la habilidad de rotación mental y la habilidad de imagen espacial de los estudiantes. Encontraron que a los estudiantes con baja capacidad de rotación de imagen les resultaba significativamente más difícil el modelo gráfico como método expositivo que a sus compañeros con alta habilidad de imagen. Cuando los estudiantes eran clasificados en función de su habilidad de imagen espacial, los resultados iban en la misma línea.

Una variable analizada en la mayoría de los estudios que incluyen medidas de imagen es el género. Por lo general, los hombres obtienen puntuaciones mayores que las mujeres en los test de rendimiento en imagen (Campos, 2014; Voyer, Voyer y Bryden, 1995). Cabe esperar que esta ventaja se pueda ver reflejada en el rendimiento en asignaturas con una fuerte carga espacial, $\mathrm{o}$ en aquellas en las que se recurra habitualmente a material visuoespacial, y de hecho este es el resultado encontrado en algunos trabajos de otros ámbitos (Reilly, Neumann y Andrews, 2017).

Teniendo en cuenta lo anterior, el objetivo de este trabajo era analizar si las habilidades de imagen y el género influían en el rendimiento académico en Economía. En concreto, se analizó si existen diferencias significativas en el rendimiento en la asignatura de Política Económica, y en una prueba sobre un modelo gráfico ampliamente utilizado en Economía, en función del género, y de dos medidas de imagen (la habilidad para formar imágenes mentales espaciales y la habilidad de rotación mental).

\section{Método}

\section{Participantes}

En la investigación participaron 119 estudiantes (51 hombres y 68 mujeres), del Grado en Administración y Dirección de Empresas (ADE) de la Facultad de Economía y Empresa de la Universidad de A Coruña, con una media de edad de 21.08 años, una desviación típica de 2.21, y un rango entre 19 y 28 años.

\section{Material}

Prueba sobre la política macroeconómica en el modelo IS-LM, consistente en cinco preguntas considerando un gráfico de cuatro cuadrantes. Cada pregunta, puntuable de 0 a 5, implica la construcción de un gráfico y su explicación. Los participantes disponen de 30 minutos para su realización. Ejemplo de pregunta: 1. Realice un gráfico de cuatro cuadrantes del modelo IS-LM orientado a estudiar los efectos de la política fiscal (IS). Analice las consecuencias sobre la renta de equilibrio, y el tipo de interés de equilibrio, de una política fiscal expansiva consistente en el aumento del gasto público.

Test que mide la habilidad para la rotación mental, el test Mental Rotation Test (MRT; Vandenberg y Kuse, 1978). Este test contiene 10 ítems, cada uno de los cuales consta de una figura criterio, dos alternativas correctas, y dos alternativas incorrectas. Las figuras correctas son semejantes al modelo en la estructura, pero están en posición diferente. Los participantes tienen 3 minutos para completar el test.

Test Measure of the Ability to Form Spatial Mental Imagery (MASMI; Campos, 2009; 2013). El test consta de un cubo descompuesto que los participantes tienen que cerrar mentalmente antes de contestar a cada una de las 23 preguntas del test. Cada pregunta tiene cuatro respuestas, dos son siempre ciertas, y dos son siempre falsas. El total de la puntuación se consigue sumando las respuestas correctas y restando las respuestas incorrectas, por lo que la puntuación total en el test puede oscilar entre $46 \mathrm{y}-46$. Los individuos disponen de 5 minutos para responder a todas las preguntas del test.

\section{Procedimiento}

A los alumnos, en sus respectivas clases, se les aplicaron los instrumentos antes mencionados, MASMI y MRT. En otra sesión se realizó la prueba sobre política macroeconómica en el modelo IS-LM y se obtuvo la puntuación para cada participante. El modelo IS-LM forma parte del programa de la asignatura de Política Económica, lo que garantizó que todo el alumnado lo conocía en el momento de realizar la prueba. Al final del cuatrimestre se recogieron las calificaciones en la asignatura de Política Económica, que constituyen la otra medida del rendimiento académico. Esta puntuación está entre 0 y 7 . Los tres puntos restantes de la calificación en la asignatura se otorgan por la asistencia y participación en las prácticas de la asignatura, no valorables en este estudio.

Se consideró como "baja habilidad de rotar imágenes" a aquellos participantes con un valor en el test MRT inferior a la media obtenida por los participantes, que fue de $8.97(S D=5.45)$ y se consideró como "alta habilidad de rotar imágenes" a aquellos estudiantes con una puntuación en el test igual o superior a esa media. Un total de 57 participantes fueron clasificados con "baja habilidad de rotar imágenes" $(M=4.14 ; S D=$ 2.38), y 62 participantes fueron clasificados con "alta habilidad de rotar imágenes" $(M=13.40 ; S D=3.25)$. Se consideró como "baja habilidad espacial" a aquellos participantes con un valor en el test MASMI inferior a la puntuación media obtenida por los participantes, que fue de $10.51(S D=8.49)$ y se consideró como "alta habilidad espacial" a aquellos estudiantes con una puntuación en el test igual o superior a esa puntuación media. Un total de 65 participantes fueron clasificados con "baja habilidad espacial" $(M=4.14 ; S D=4.5)$, y 54 participantes fueron clasificados con "alta habilidad espacial" $(M=18.19 ; S D=5.11)$. 


\section{Resultados}

Con el fin de averiguar la influencia que tienen la habilidad de rotar imágenes, y el género, en el rendimiento en la prueba sobre el modelo gráfico citado, se llevó a cabo un ANOVA de 2 (género) x 2 (habilidad de imagen espacial, medida a través del MRT), tomando como variable dependiente la puntuación en la prueba de gráficos. Las medias y desviaciones típicas del Rendimiento Académico en la prueba de gráficos, obtenidas por las Mujeres y los Hombres, y Altos y Bajos en el MRT, figuran en la Tabla 1.

Tabla 1.

Medias y Desviaciones Típicas del Rendimiento Académico en la Prueba de Gráficos (Modelo IS-LM) y en la Asignatura de Política Económica, Obtenidas por las Mujeres y los Hombres Altos y Bajos en el MASMI y Altos y Bajos en el MRT

\begin{tabular}{lcccc}
\hline & \multicolumn{2}{c}{ Prueba } & Gráficos & \multicolumn{2}{c}{ Rend. Asignatura } \\
\cline { 2 - 5 } Grupos & $M$ & $S D$ & $M$ & $S D$ \\
\hline Altos MASMI & 2.08 & 1.14 & 1.95 & 1.48 \\
Bajos MASMI & 1.58 & 1.47 & 1.57 & 1.07 \\
Altos MRT & 2.02 & 1.27 & 1.94 & 1.39 \\
Bajos MRT & 1.58 & 1.40 & 1.54 & 1.12 \\
Mujeres & 2.01 & 1.40 & 1.92 & 1.31 \\
Hombres & 1.51 & 1.24 & 1.51 & 1.19 \\
Total & 1.80 & 1.35 & 1.74 & 1.27 \\
\hline
\end{tabular}

El análisis de datos muestra que el alumnado con alta habilidad de rotación de imágenes obtuvo una puntuación en la prueba de gráficos $(M=2.02 ; S D=$ 1.27) significativamente superior a la obtenida por el alumnado con baja habilidad de rotar imágenes $(M=$ $1.58 ; S D=1.40), F(1,115)=4.84, p=.03$. Las mujeres obtuvieron una puntuación en la prueba de gráficos $(M$ $=2.01 ; S D=1.40)$ significativamente superior a la obtenida por los hombres $(M=1.51 ; S D=1.24), F(1$, $115)=6.13, p=.02$. La interacción entre las dos variables, habilidad de rotación de imágenes y género, no resultó significativa, $F(1,115)=.39, p=.54$.

Para averiguar la influencia de la habilidad espacial (medida a través del MASMI) en el rendimiento en la prueba de gráficos, se efectuó una prueba $t$, tomando como variable dependiente la puntuación en la prueba de gráficos. Las medias y desviaciones típicas del rendimiento obtenido en la prueba de gráficos por Altos y Bajos en el MASMI, figuran también en la Tabla 1. Encontramos que los estudiantes con alta habilidad espacial obtuvieron en la prueba de gráficos $(M=2.08$; $S D=1.14$ ) una puntuación significativamente superior a la de los estudiantes con baja habilidad de imagen espacial $(M=1.58 ; S D=1.47), t(117)=2.03, p=.04$.

Otro de los objetivos del trabajo era analizar la influencia que tienen la habilidad de rotar imágenes y el género, en el rendimiento académico en la materia de Política Económica. Para ello, se llevó a cabo un ANOVA de 2 (género) x 2 (habilidad de rotar imágenes, medida a través del MRT), tomando como variable dependiente la calificación en la asignatura de Política Económica. Las medias y desviaciones típicas del Rendimiento Académico en la asignatura de Política Económica, obtenidas por las Mujeres y los Hombres,
Altos y Bajos en el MRT, figuran en la Tabla 1. El análisis de datos muestra que el alumnado con alta habilidad de rotación de imágenes obtuvo una calificación en la asignatura de Política Económica $(M=$ $1.94 ; S D=1.39$ ) significativamente superior a la obtenida por el alumnado con baja habilidad de rotar imágenes $(M=1.54 ; S D=1.12), F(1,115)=4.59, p=$ .03. Las mujeres obtuvieron una puntuación en la asignatura $(M=1.92 ; S D=1.31)$ significativamente superior a la obtenida por los hombres $(M=1.51$; $S D=$ 1.19), $F(1,115)=4.73, p=.03$. La interacción entre las dos variables, habilidad de rotación de imágenes y género, no resultó significativa, $F(1,115)=.02, \quad p=$ .90 .

A continuación, para averiguar la influencia que tiene la habilidad espacial (medida a través del MASMI) en el rendimiento académico en la materia de Política Económica, se efectuó una prueba $t$, tomando como variable dependiente la calificación en la asignatura de Política Económica. Las medias y desviaciones típicas del rendimiento obtenido en la asignatura de Política Económica por Altos y Bajos en el MASMI, figuran en la Tabla 1. No se encontraron diferencias significativas en el rendimiento a académico de los estudiantes Altos y Bajos en habilidad de imagen espacial, $t(117)=1.63$, $p=.11)$.

\section{Discusión}

Las puntuaciones que obtuvieron los participantes con alta habilidad de rotación mental en la prueba de gráficos y en la materia evaluada (las dos medidas de rendimiento utilizadas en este estudio), fueron significativamente superiores a las de los participantes con baja habilidad de rotación. También la puntuación que obtuvieron los estudiantes con alta habilidad para formar imágenes espaciales en la prueba de gráficos fue significativamente superior a la obtenida por los estudiantes con baja habilidad espacial. Ambos resultados están en la línea de lo hallado en otros ámbitos (Cui y cols., 2017; Guillot y cols., 2007; Yoon y Min, 2016), en los que también se encontró una relación entre habilidad espacial y habilidad de rotación de imagen con algunos aspectos del rendimiento, y son coherentes con los resultados del trabajo de Dopico, González y Campos-Juanatey (en prensa) (menor percepción de dificultad con el modelo gráfico en los estudiantes con alta capacidad de rotación mental y en los de alta habilidad de imagen espacial).

Cohn y cols. (2001) no encontraron diferencias en el rendimiento de los estudiantes a los que se les presentaron gráficos en sus clases de Economía, en comparación con aquellos a los que no se les presentaban gráficos. Sin embargo, como los autores reconocen, ciertos controles ausentes en sus estudios pueden explicar ese resultado. En este trabajo se garantizó que todos los participantes conocieran el modelo gráfico evaluado.

Otro resultado a destacar es que las dos puntuaciones de rendimiento estudiadas fueron significativamente más altas en las mujeres que en los hombres. Ambas puntuaciones de rendimiento intentaban reflejar el 
desempeño de los sujetos con material gráfico, contenido espacial que, basándose en la literatura sobre el tema, permitía esperar una rendimiento mayor en los hombres (Reilly, Neumann y Andrews, 2017). Sin embargo, tal y como se ha descrito en el procedimiento, la puntuación en la prueba sobre gráficos recogía aspectos complementarios a la comprensión del gráfico, como su explicación, orden y claridad expositiva, etc. Lo mismo cabe decir de la parte calificable de la asignatura evaluada, que reflejaba contenidos de la materia, utilización de la terminología económica, de nuevo claridad expositiva, etc. Se necesitan nuevas investigaciones que profundicen en el análisis de la interpretación que realizan los estudiantes de gráficos específicos, para contribuir a aclarar el papel de las variables analizadas en este estudio.

\section{Referencias}

Campos, A. (2009). Spatial imagery: A new measure of the visualization factor. Imagination, Cognition and Personality, 29, 31-39.

Campos, A. (2013). Reliability and percentiles of a measure of spatial imagery. Imagination, Cognition and Personality, 32, 427-431. htpp://dx.doi: 10.2190/IC.32.4.f

Campos, A. (2014). Gender differences in imagery. Personality and Individual Differences, 59, 107-111. http://dx.doi.org/10.1016/j.paid.2013.12.010

Cohn, E., Cohn, S., Balch, D. C., y Bradley, J. (2001). Do graphs promote learning in principles of Economics? Journal of Economic Education, 32, 4, 299-310.

Cui, D., Wilson, T. D., Rockhold, R. W., Lehman, M. N., y Lynch, J. C. (2017). Evaluation of the effectiveness of 3D vascular stereoscopic models in anatomy instruction for first year medical students. Anatomical Sciences Education, 10, 34-45. htpp://dx.doi: 10.1002/ase.1626. E

Dopico, J. A., González, M. A., y Campos-Juanatey, D. (en prensa). Habilidades de imagen y valoración de los métodos explicativos en Economía. Actas del XIV Congreso Internacional Galego-Portugués de Psicopedagoxía. Braga.

Guillot, A., Champely, S., Batier, C., Thiriet, P. y Collet, C. (2007). Relationship between spatial abilities, mental rotation and functional anatomy learning. Advances in Health Sciences Education, 12, 491-507. htpp://dx.doi:10.1007/s10459-006-9021-7

Khine, M. S. (Ed.). (2017). Visual-spatial Ability in STEM Education. Transforming Research into Practice. New York: Springer.

Reilly, D., Neumann, D. L., y Andrews, G. (2017). Gender differences in spatial ability: Implications for STEM education and approaches to reducing the gender gap for parents and educators. En M. S. Khine (Ed.), Visual-spatial Ability in STEM Education. Transforming Research into Practice (pp. 195-224). New York: Springer.

Reingewertz, Y. (2013). Teaching macroeconomics through flowcharts. International Review of
Economics Education, 14, 86-93. http://dx.doi.org/10.2139/ssrn.2200023

Vanderberg, S. G., y Kuse, A. R. (1978). Mental rotations, a group test of three-dimensional spatial visualization. Perceptual and Motor Skills, 47, 599604.

Voyer, D., Voyer, S., y Bryden, M. P. (1995). Magnitude of sex differences in spatial abilities: A meta-analysis and consideration of critical variables. Psychological Bulletin, 117, 250-270. htpp://dx.doi:10.1037/0033-2909.117.2.250

Yoon, S. Y. (2001). Psychometric properties of the Revised Purdue Spatial Visualization Tests: Visualization of Rotations (The Revised PSVT: R) (Tesis Doctoral). Purdue University, West Lafayette, Indiana.

Yoon, S. Y. y Min, K.-H. (2016). College students' performance in an introductory atmospheric science course: Associations with spatial ability. Meteorological Applications, 23, 409-419. htpp://dx.doi: 10.1002/met.1565 